\title{
Experimental and Numerical Analysis of Air Trapping in a Porous Medium with Coarse Textured Inclusions
}

\author{
Paulina SZYMAŃSKA ${ }^{1}$, Witold TISLER ${ }^{1}$, Cindi SCHÜTZ ${ }^{2}$, \\ Adam SZYMKIEWICZ ${ }^{1}$, Insa NEUWEILER ${ }^{2}$, and Rainer HELMIG ${ }^{3}$ \\ ${ }^{1}$ Gdańsk University of Technology, Faculty of Civil and Environmental \\ Engineering, Department of Geotechnics, Geology and Marine Civil Engineering, \\ Gdańsk, Poland; e-mail: adams@pg.gda.pl (corresponding author) \\ ${ }^{2}$ Institute of Fluid Mechanics and Environmental Physics in Civil Engineering, \\ Leibniz University Hannover, Hannover, Germany \\ ${ }^{3}$ Institute for Modelling of Hydraulic and Environmental Systems, \\ University of Stuttgart, Stuttgart, Germany
}

\begin{abstract}
The paper presents a 2D upward infiltration experiment performed on a model porous medium consisting of fine sand background with two inclusions made of coarser sands. The purpose of the experiment was to investigate the effects of structural air trapping, which occurs during infiltration as a result of heterogeneous material structure. The experiment shows that a significant amount of air becomes trapped in each of the inclusions. Numerical simulations were carried out using the two-phase water-air flow model and the Richards equation. The experimental results can be reproduced with good accuracy only using a two-phase flow model, which accounts for both structural and pore-scale trapping. On the other hand, the Richards equation was not able to represent the structural trapping caused by material heterogeneity.
\end{abstract}

Key words: vadose zone, Richards equation, heterogeneous soils, air trapping.

Ownership: Institute of Geophysics, Polish Academy of Sciences;

(c) 2016 Szymańska et al. This is an open access article distributed under the Creative Commons Attribution-NonCommercial-NoDerivs license,

http://creativecommons.org/licenses/by-nc-nd/3.0/. 


\section{INTRODUCTION}

The pore space of soils and rocks in the vadose zone is filled partly with water and partly with air. In many practical problems, the focus is primarily on the flow of water and the air flow is neglected. This approach is justified by assuming that the pore air is continuous, connected to the atmospheric air and much more mobile than the pore water. This implies that any perturbation of the air pressure instantly equilibrates with the atmosphere and the pore air remains essentially at constant atmospheric pressure. In such a case the water flow can be described by a single equation, known as the Richards equation (RE) (Richards 1931). In contrast, if the above assumptions are not satisfied, one should use a full two phase model (2PH), which accounts explicitly for the flow of both water and air, caused by potential gradients within each phase (e.g., Helmig 1997).

While the RE is commonly used in hydrological and geotechnical modeling, a number of authors showed its limitations caused by various factors. First, the mobility ratio between air and water is not infinite and even at medium water saturation levels there are some discrepancies in the results obtained with RE and 2PH approaches, e.g., Tegnander (2001). Close to full water saturation, the smaller viscosity of air is offset by its smaller relative permeability, which makes the mobilities of the two fluids similar. In such a case, important differences between the models can be observed (Forsyth 1988). Other well known situations where RE is not applicable concern infiltration in laboratory columns with sealed bottom (Vachaud et al. 1973, Touma et al. 1984, Touma and Vauclin 1986), ponded infiltration over large area (Hammecker et al. 2003, Delfs et al. 2013) or overtopping of earth dikes by flood wave (Bogacz et al. 2006, Leśniewska et al. 2008).

Attention has been also directed to the impact of heterogeneous soil structure on the patterns of air and water flow. In this context the importance of the so-called air-entry barriers has been emphasized (Silliman et al. 2002). They are regions of fine textured material, remaining fully or nearly fully water-saturated even at significant negative water pressures, until their characteristic value of the air entry pressure is exceeded. Experiments in a twodimensional flume described in (Silliman et al. 2002) showed that thin layers or inclusions of coarse material present in the capillary fringe did not drain due to the presence of quasi saturated fine material around them, and provided important paths for horizontal tracer migration. Other experiments (Kuang et al. 2011) showed that drainage from a laboratory column filled with coarse sand is significantly hampered by the presence of a thin layer of fine sand at the upper surface of sand. The fine layer remained quasisaturated with water and consequently had very low permeability to air, causing retarded drainage, while the suction in the coarse sand remained 
higher than it would result from the water retention curve. Several contributions focused on heterogeneity patterns consisting of coarse textured inclusions embedded in fine textured background (Dunn and Silliman 2003, Dunn 2005, Vasin et al. 2008, Szymkiewicz et al. 2012, 2014; Haberer et al. 2015). If the inclusions are disconnected from each other and from the atmospheric air, two types of interesting phenomena can be observed. First, if the heterogeneous medium is subject to drainage starting from full water saturation, the inclusions remain saturated until the suction exceeds the entry pressure of the fine background material, which can be much larger than their own entry pressure. Second, during imbibition the background material becomes fully or quasi fully saturated at relatively large negative pressure, corresponding to a much lower saturation in the coarse material. However, once the pores of the background material are completely filled with water, the air from coarse inclusions cannot escape. Even if the water pressure in the system becomes positive (larger than atmospheric), the inclusions remain unsaturated. Both types of behavior were confirmed by laboratory experiments and numerical analyses (Dunn and Silliman 2003, Dunn 2005, Vasin et al. 2008). Such effects were also investigated for other types of two phase flow, e.g., in water-NAPL or water-CO2 systems (Dunn 2005, Szymkiewicz et al. 2011, Saadatpoor et al. 2009). If the heterogeneous medium contains a large number of inclusions, it is possible to derive an upscaled model with effective parameters, which is characterized by hysteresis of the water retention function and the phase permeability functions (Mikelic et al. 2002, van Duijn et al. 2007, Schweizer 2008, Szymkiewicz et al. 2011, 2012; Szymkiewicz 2013). One should also note that the effects occurring on coarse-fine soil interfaces are used in capillary barriers protecting waste storage site from infiltration or in the drainage layers placed under lawns. These structures are composed of a fine-textured soil layer supporting vegetation with underlying coarse grained material. In unsaturated conditions the coarse material has very low permeability, which effectively limits the downward infiltration. Only if the water pressure at the interface reaches values close to zero, a significant amount of water can enter the coarse material and is rapidly transmitted by this layer. Capillary barriers were a subject of numerous modeling studies (e.g., Oldenburg and Pruess 1993, Aubertin et al. 2009, Prédélus et al. 2015). Assuming that the air in coarse layer can move unobstructed downward and laterally, the Richards' equation can be used with sufficient accuracy for such analyses (Webb 1998).

The entrapment of air in coarse inclusions during imbibition, which will be called here structural trapping, is a phenomenon qualitatively distinct from the pore-scale entrapment of isolated air bubbles. The latter process is well recognized in the literature and has been a subject of several studies, e.g., Marinas et al. (2013), McLeod et al. (2015). Air bubbles in pores typi- 
cally arise during infiltration, but may be also products of various biological and chemical processes in porous medium. In order to account for their presence, the concept of "field-saturated" conditions is often used in vadose zone modeling. The maximum attainable value of the volumetric water content $\theta_{s w}$ is smaller than the porosity $\phi$. The residual air saturation varies in a wide range, from 4 to $54 \%$ of the soil porosity (Marinas et al. 2013). Consequently, the field-saturated hydraulic conductivity is also smaller than the "true" saturated conductivity, even up to 10 times (Marinas et al. 2013).

In natural soils we can expect overlapped effects of structural and porescale air trapping, occurring especially in the zone of water table fluctuations and the adjacent capillary fringe. This transition zone, termed "partially saturated fringe" (Berkowitz et al. 2004) is increasingly recognized as crucial for contaminant transport and various biochemical processes in soils (Berkowitz et al. 2004, Silliman et al. 2002, Yakirevich et al. 2010), since the entrapped air determines oxygen availability (Haberer et al. 2015). More research is needed to better understand the air and water dynamics in the vicinity of the water table. This paper presents the results of a laboratory experiment on a model porous medium with separated coarse inclusions, subject to upward infiltration. We focus on the combined effects of structural and pore scale trapping. Moreover, we compare numerical solutions obtained with the Richards equation and the two phase flow model. To the best of our knowledge, such a comparison of RE and $2 \mathrm{PH}$ models with experimental results for heterogeneous media has not been undertaken before, since in previous contributions only one of those two approaches was used, e.g., $2 \mathrm{PH}$ model in (Dunn 2005) or RE in (Vasin et al. 2008).

\section{EXPERIMENTS}

Experiments were carried out in the laboratory of the Institute of Fluid Mechanics and Environmental Physics in Civil Engineering, Leibniz University, Hannover (Szymańska 2012). The main part of the experimental setup was a glass flume having dimensions $55 \mathrm{~cm}$ by $29 \mathrm{~cm}$ by $1.2 \mathrm{~cm}$, which allowed to impose conditions of two-dimensional flow. Three types of well sorted sand were used, which will be denoted here as fine, medium and coarse (see Table 1). Fine sand was used as the background material, filling most of the flume, while medium and coarse sands formed two inclusions placed symmetrically (Fig. 1). The purpose of the experiment was to investigate the air trapping in inclusions during upward infiltration. Water entered the flume from two inlets located at the bottom of the device.

In order to facilitate observation of the changing saturation field, water was dyed to a blue colour. During the experiment, pictures of the flume were taken by a camera placed in front of it. The saturation field was obtained by 
Table 1

Parameters of three sands used in the experiment

\begin{tabular}{|c|c|c|c|c|}
\hline Parameter & Unit & Coarse & Medium & Fine \\
\hline$d$ & {$[\mathrm{~mm}]$} & 0.7 to 1.2 & 0.4 to 0.8 & 0.1 to 0.3 \\
$\phi$ & {$[-]$} & 0.540 & 0.450 & 0.370 \\
\hline \multicolumn{5}{|c|}{ MVG - measurements } \\
\hline$\theta_{s w}$ & {$[-]$} & - & 0.360 & 0.280 \\
$\theta_{r w}$ & {$[-]$} & 0.00 & 0.00 & 0.02 \\
$p_{g ; d}$ & {$[\mathrm{~Pa}]$} & 1143 & 1990 & 4004 \\
$p_{g, i}$ & {$[\mathrm{~Pa}]$} & 565 & - & - \\
$n$ & {$[-]$} & 6.07 & 4.86 & 3.35 \\
$k_{s}$ & {$\left[\mathrm{~m}^{2}\right]$} & $1.57 \cdot 10^{-11}$ & $8.66 \cdot 10^{-12}$ & $4.48 \cdot 10^{-12}$ \\
\hline \multicolumn{5}{|c|}{ BCB0- first estimation } \\
\hline$\theta_{s w}$ & {$[-]$} & 0.540 & 0.450 & 0.370 \\
$\theta_{r w}$ & {$[-]$} & 0.00 & 0.00 & 0.00 \\
$p_{e ; i}$ & {$[\mathrm{~Pa}]$} & 388 & 746 & 1582 \\
$\lambda$ & {$[-]$} & 2.86 & 2.25 & 1.47 \\
$k_{s}$ & {$\left[\mathrm{~m}^{2}\right]$} & $1.57 \cdot 10^{-11}$ & $8.66 \cdot 10^{-12}$ & $4.48 \cdot 10^{-12}$ \\
\hline \multicolumn{5}{|c|}{ BCB1 - best fit } \\
\hline$\theta_{s w}$ & {$[-]$} & 0.443 & 0.370 & 0.355 \\
$\theta_{r w}$ & {$[-]$} & 0.00 & 0.00 & 0.00 \\
$p_{e ; i}$ & {$[\mathrm{~Pa}]$} & 300 & 500 & 1100 \\
$\lambda$ & {$[-]$} & 4.0 & 4.0 & 2.8 \\
$k_{s}$ & {$\left[\mathrm{~m}^{2}\right]$} & $2.56 \cdot 10^{-11}$ & $1.86 \cdot 10^{-11}$ & $1.44 \cdot 10^{-11}$ \\
\hline
\end{tabular}

image analysis techniques. A digital camera (Nikon D90) was placed in front of the experimental device. Still pictures were taken in $10 \mathrm{~s}$ time intervals. The resolution of the pictures was 4288 by 2848 pixels. During image processing, the number of pixels representing dyed water was used to obtain the average saturation in each of the three materials. The reference level of saturation was obtained from an independent experiment, where the flume was filled with sands forming three vertical strips parallel to each other, and having the same porosity as in the main experiment. In contrast to the principal experiment, the device was flushed with $\mathrm{CO}_{2}$ and slowly saturated by upward water infiltration. In such conditions, no structural trapping could occur and the pore trapping was negligibly small, so it was assumed that the image parameters correspond to full saturation in each material. The total volume of water in the flume was monitored with a balance. 

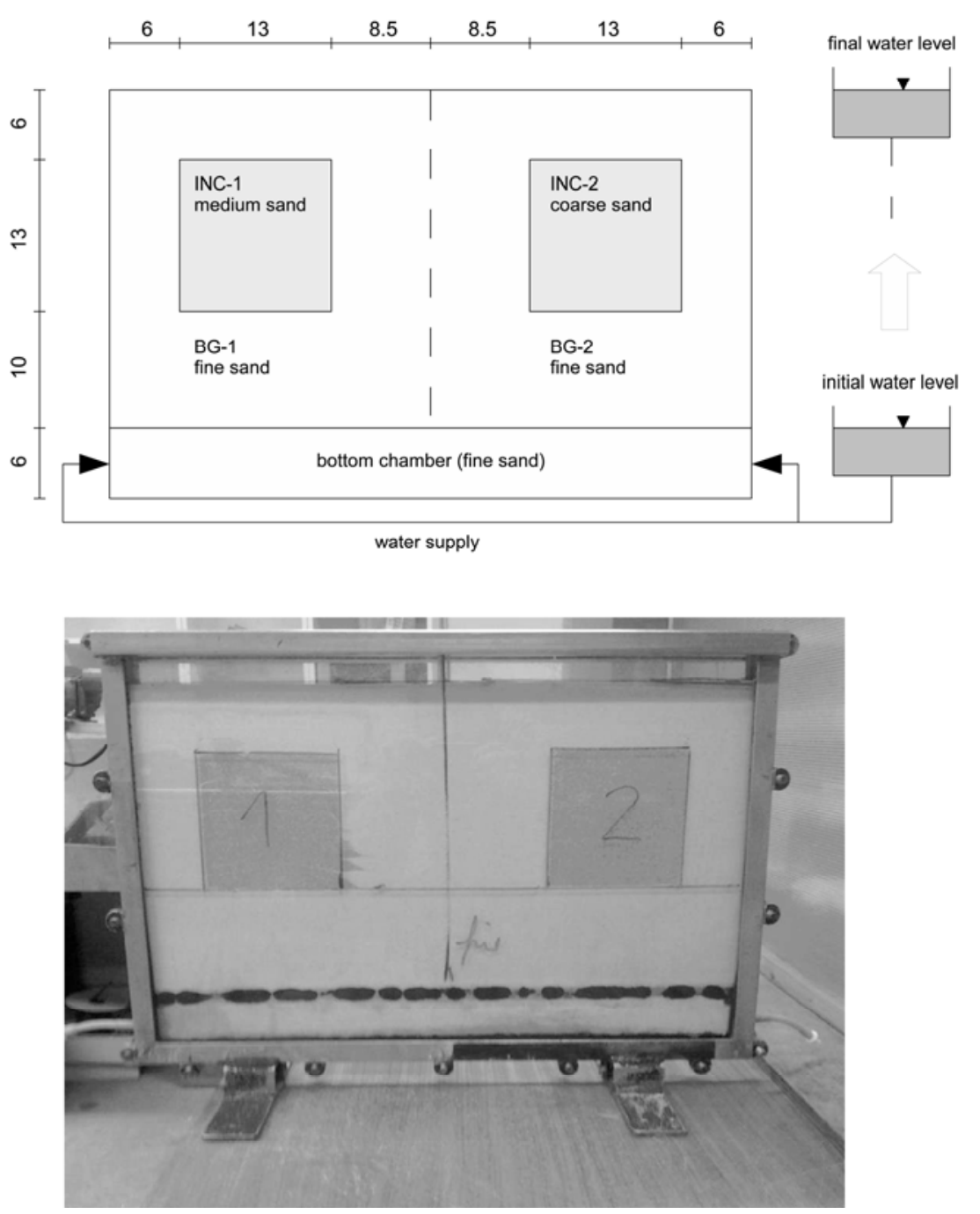

Fig. 1. Scheme of the experimental setup (top) and picture of the flume after packing (bottom). Dimensions in $\mathrm{cm}$.

Initially all three sands were in air dry conditions. After filling the flume, the inlets were connected to a water-filled reservoir with water level at the same elevation as the bottom of the flume. The water level in reservoir was kept constant for 12 hours, in order to approach the state of capillary equilibrium. During this time, capillary forces caused significant increase of saturation in the fine material; however, the two inclusions remained virtually dry (Fig. 2, $t=0$ ). 

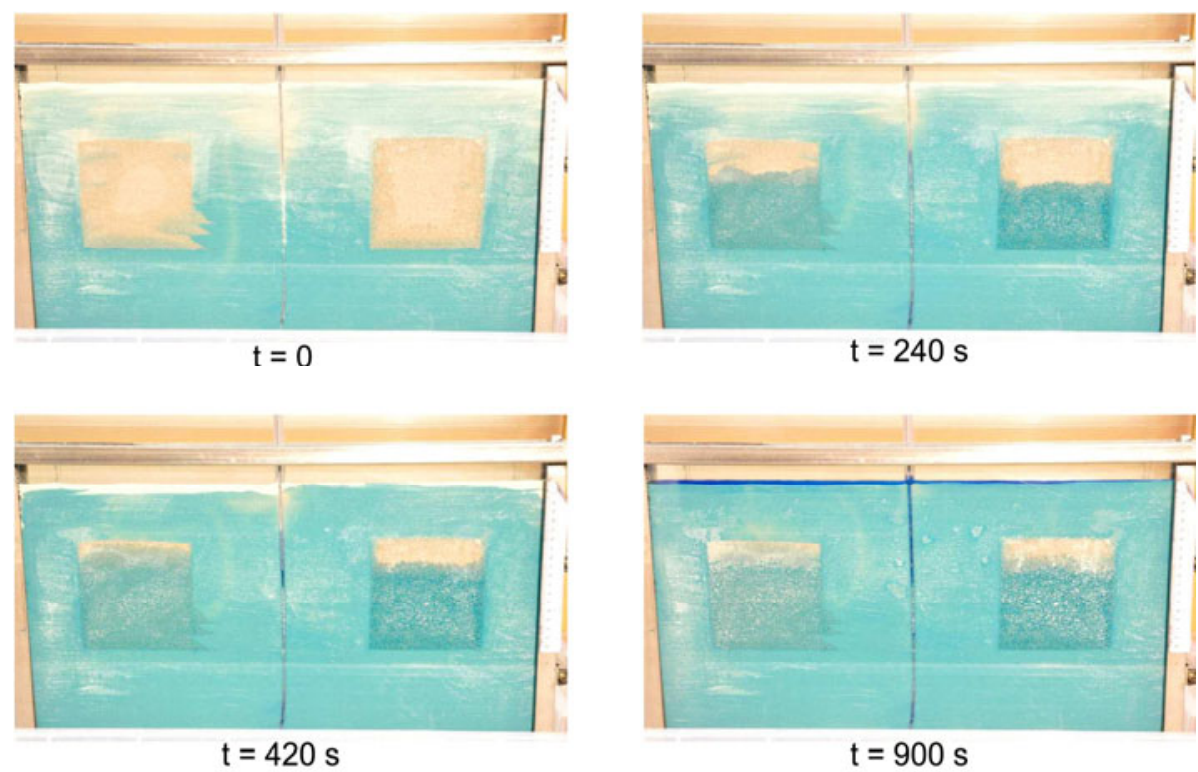

Fig. 2. Distribution of dyed water in the flume at four stages of the experiment.

The actual experiment was started by rising the reservoir with water in such a way that the water level corresponded to the upper surface of the fine sand in the flume. The rising took about $10 \mathrm{~s}$. The resulting increase of positive water pressure at the bottom of the flume caused upward infiltration. Water distribution at various stages of infiltration is shown in Fig. $2(t=240$, 420 , and $900 \mathrm{~s}$ ). A stable configuration of water distribution was achieved about $600 \mathrm{~s}$ after the rising of the water table started. The observations were continued for another $25 \mathrm{~min}$, without any visible changes in the saturation distribution inside the sand. At about $900 \mathrm{~s}$ a thin layer of free water appeared at the surface of fine sand, indicating that equilibrium with supplying water reservoir had been reached.

From Fig. 2 it can be clearly seen that the upward movement of water occurs principally in fine sand, where capillary forces are relatively strong. The saturation process in the inclusions starts from the bottom, where the water pressure is high enough to ensure sufficient hydraulic conductivity to enter the coarse porous medium. A clear horizontal boundary can be seen between the saturated and dry zone in the inclusions. There is apparently no flow into the inclusions from the upper part of their perimeter, even though they contact fine sand at relatively high saturation. Only in the later stages of infiltration, the boundary between dry and wet zone becomes more diffuse. The inclusions remain only partly saturated till the end of the experiment. 


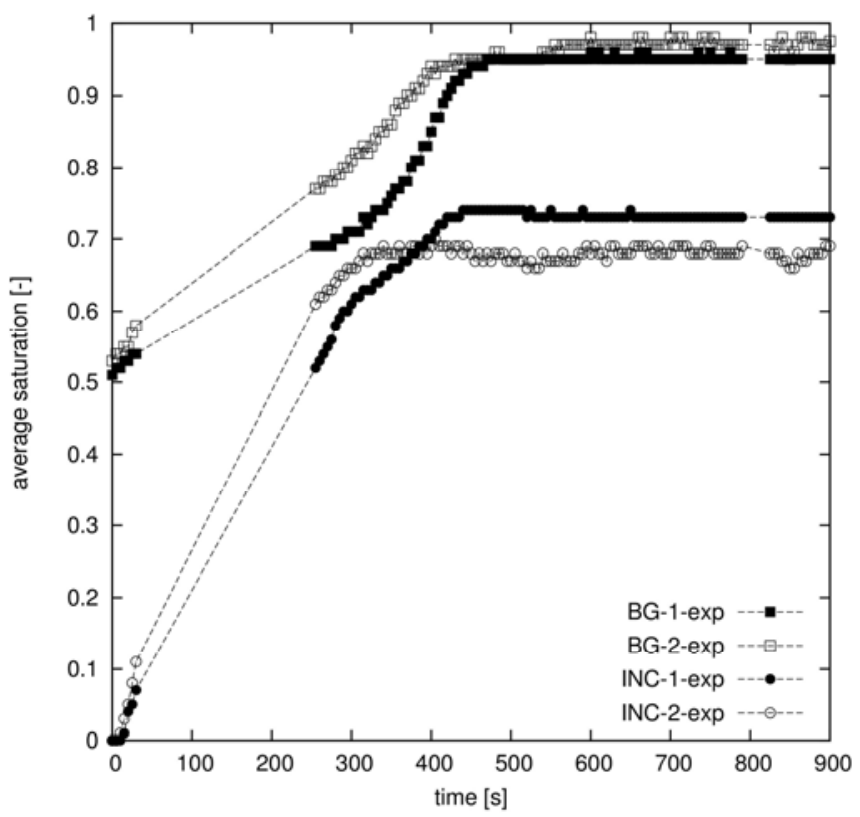

Fig. 3. Evolution of average water saturation in four regions of the flume during the experiment, as obtained by image analysis.

One can clearly see an unsaturated region at the top of each inclusion, and also many small air bubbles in the lower parts of inclusions. This indicates the occurrence of both structural trapping and pore scale trapping.

Figure 3 presents plots of the average saturation evolution in time, as obtained from the image analysis. There are four average values, corresponding to four regions of the plume. The fine sand background was divided into two regions, the left-hand one, BG1, around the medium sand inclusion INC1, and the right-hand one, BG2, around the coarse sand inclusion INC2. These plots confirm visual observations. It can be seen that at $t=0$, when the medium is in a state close to hydrostatic equilibrium, the average saturation in the fine sand is close to 0.5 , while the saturation in inclusions is practically zero. After the rising of the water table saturation in all regions increases, with higher increase rate in inclusions than in the background material. Neither the background nor inclusions become fully saturated during the experiment, however the background material becomes very close to full saturation, with the maximum saturation value about 0.97 . On the other hand, the final values of saturation in inclusions are 0.73 for the medium sand and 0.69 for the coarse sand. These are average values, which account for both structural and pore-scale trapping. Starting from about $600 \mathrm{~s}$, a stable state of saturation is reached, which lasts till the end of the experiment. 


\section{NUMERICAL MODELING}

The two phase flow model used in this paper is based on the following assumptions: the fluids are immiscible (due to the short time of the experiments the dissolution of air in water is not modelled); the flow is isothermal; each porous material is rigid and has isotropic permeability. Under these assumptions the governing equations for either water or air, representing the mass balance principle for each fluid, can be written as follows (e.g., Helmig 1997):

$$
\frac{\partial}{\partial t}\left(\rho_{\alpha} \phi S_{\alpha}\right)+\nabla \cdot\left(\rho_{\alpha} \mathbf{v}_{\alpha}\right)=0,
$$

where $\alpha$ is the fluid phase index ( $a=$ air, $w=$ water), $\rho_{\alpha}$ the fluid density, $\phi$ the porosity of the medium, $S_{\alpha}$ the fluid saturation and $\mathbf{v}_{\alpha}$ the volumetric fluid flux (or seepage velocity). According to the Darcy's law for isotropic medium the flux can be defined as:

$$
\mathbf{v}_{\alpha}=-\frac{k_{s} k_{r \alpha}}{\mu_{\alpha}}\left(\nabla p_{\alpha}-\rho_{\alpha} \mathbf{g}\right),
$$

where $k_{s}$ is the intrinsic permeability, $k_{r \alpha}$ the relative permeability for phase $\alpha, \mu_{\alpha}$ fluid viscosity, $p_{\alpha}$ fluid pressure (or, more generally, pressure potential), and $\mathbf{g}$ the gravitational acceleration vector.

The governing equations have to be completed by additional relationships. The difference in pressure potentials between air and water is known as the capillary pressure or suction and is related to the water saturation via the water retention function. Moreover, the relative permeabilities depend on the fluid saturations. These interdependencies are commonly described using the Mualem-van Genuchten (MVG) model (Mualem 1976, van Genuchten 1980):

$$
\begin{gathered}
S_{e}=\left[1+\left(\frac{p_{c}}{p_{g}}\right)^{n}\right]^{-m} \\
k_{r w}=\sqrt{S_{e}}\left[1-\left(1-S_{e}^{1 / m}\right)^{m}\right]^{2} \\
k_{r a}=\sqrt{1-S_{e}}\left(1-S_{e}^{1 / m}\right)^{2 m}
\end{gathered}
$$

where $S_{e}$ is the effective water saturation, $p_{c}$ the capillary pressure or suction $\left(p_{c}=p_{a}-p_{w}\right), p_{g}$ the pressure scaling parameter, and $n, m$ are shape parameters $(m=1-1 / n)$. The effective water saturation represents values of saturation normalized between residual states of water and air: 


$$
S_{e}=\frac{S_{w}-S_{r w}}{1-S_{r w}-S_{r a}}=\frac{\theta_{w}-\theta_{r w}}{\theta_{s w}-\theta_{r w}}
$$

where $S_{r w}$ and $S_{r a}$ are residual saturations of water and air, respectively, $\theta_{w}$ is the volumetric water content, $\theta_{r w}$ the residual water saturation, and $\theta_{s w}$ is the water content at apparent saturation, with air phase at residual state.

Another widely used set of functions is the Brooks-Corey-Burdine (BCB) model (Brooks and Corey 1964, Burdine 1953):

$$
\begin{gathered}
S_{e}=\left\{\begin{array}{l}
\left(\frac{p_{c}}{p_{e}}\right)^{\lambda} \text { if } \quad p_{c}>p_{e} \\
1 \quad \text { if } \quad p_{c} \leq p_{e}
\end{array}\right. \\
k_{r w}=S_{e}^{3+2 / \lambda} \\
k_{r a}=\left(1-S_{e}\right)^{2}\left(1-S_{e}^{1+2 / \lambda}\right)
\end{gathered}
$$

where $p_{e}$ is the air-entry pressure, and $\lambda$ is the shape parameter. In the BCB model the air entry effect is explicitly represented, i.e., the porous material remains saturated with water until a specific value of the capillary pressure is exceeded. In the MVG model the air entry pressure is not accounted for, although for large values of $n$ the effective water saturation remains close to 1 for a range of positive capillary pressure values. The following relationships between parameters of the two models have been suggested (Lenhard et al. 1989):

$$
\begin{gathered}
\lambda=(n-1)\left(1-0.5^{n /(n-1)}\right) \\
p_{e}=p_{g} \varsigma^{1 / \lambda}\left(\varsigma^{n /(n-1)}-1\right)^{1 / n} \\
\varsigma=0.72-0.35 \exp \left(-n^{4}\right)
\end{gathered}
$$

The Richards' equation (Richards 1931) represents a special case of twophase model, obtained for the assumption of constant air pressure. In such a case the capillary pressure is uniquely defined by the water pressure and the relative permeability and water saturation depend only on the water pressure. Mass balance is considered only for the water phase.

In order to facilitate comparisons, all numerical simulations described in this paper and the following sections were carried out using the same approach to discretize $2 \mathrm{PH}$ and $\mathrm{RE}$ mathematical models. The algorithm is based on vertex centered finite volume discretization in space and fully implicit first-order finite difference discretization in time. For each mathemati- 
cal model the discretization results in a system of nonlinear algebraic equations, which must be solved with respect to the primary unknowns. These unknowns represent the nodal values of the water pressure, and additionally for $2 \mathrm{PH}$ also nodal values of the water saturation. The grid consisted of uniform square cells, $1 \mathrm{~cm}$ by $1 \mathrm{~cm}$. The computational nodes correspond to grid vertices, while the material properties are assigned to grid cells since the material properties are assigned to grid cells and not to vertices (nodes), the water saturation was discontinuous at vertices positioned on inclusion boundaries. For these nodes, as the primary variable we chose the saturation from the material with the lowest value of the entry pressure and we calculate the saturation in the other material from the extended capillary equilibrium condition (de Neef and Molenaar 1997). Further details of the algorithm can be found in Szymkiewicz (2013).

In all simulations the same initial and boundary conditions were used. As the initial condition we assumed a uniform value of air pressure ( $p_{a}$ atmospheric) and hydrostatic distribution of water pressure, with $p_{w}$ at the initial position of the water table (Fig. 1). The boundary condition at the bottom was given in terms of the water pressure calculated from the position of the water table in the supplying reservoir. The value increased from the initial one to the final one within $10 \mathrm{~s}$ and then was kept constant till the end of the simulations. The water saturation at the bottom was assumed to be $S_{w}=1$ for the whole duration of the experiment. These values of water pressure and saturation were applied along the whole bottom edge of the flume, even though in reality the water was supplied from two inlets located at the sides of flume just above the bottom. However, visual examination shows a rather uniform upward movement of the infiltration front in fine sand (Fig. 2), which justifies the application of a uniform boundary condition along the bottom of the flume. At the top of the flume a no-flow condition was specified for water, while air was kept at a constant atmospheric pressure. The vertical sides of the flume were modeled as impermeable to both water and air.

\section{INITIAL ESTIMATION OF MATERIAL PARAMETERS}

In order to obtain hydraulic characteristics of the three sands, experiments were carried out at the Helmholtz Centre for Environmental Research UFZ. The retention curve for each sand was obtained from multistep outflow experiments, starting from fully saturated conditions. Additionally, the saturated hydraulic conductivity of each sand was also measured. The parameters of MVG model were fitted to the results of the outflow experiment. The obtained values are listed in Table 1 (denoted by MVG model - measurements). 
The parameters obtained in drainage (outflow) experiments cannot be used directly in infiltration experiments, due to hysteresis of the water retention curve (e.g., Luckner et al. 1989). Also, the samples of sand used in the independent experiments were more compacted than the sand in 2D flume, as indicated by the comparison of $\theta_{s w}$ for MVG with porosity $\phi$ in Table 1 (the outflow experiments started with full saturation, so $\theta_{s w}$ corresponds to the porosity in this case). It is well known that the porosity and packing method significantly affects hydraulic parameters of soils.

For our preliminary numerical simulations we derived a set of hydraulic parameters based on the independent experiments described above and the following assumptions:

व The MVG water retention functions for imbibition are characterized by the same values of the parameter $n$ as for drainage, while the values of the pressure scaling parameter $p_{g}$ are two times smaller. The latter assumption is supported by the literature (Luckner et al. 1989, Likos et al. 2014) and by measurements of the water retention curve in imbibition conditions for the coarse sand, which yielded $p_{g, i}=565 \mathrm{~Pa}$, i.e., nearly exactly $p_{g ; d} / 2$.

- In order to account explicitly for the air entry pressure, the water retention functions described by MVG model are converted to functions described by BCB model, according to Eqs. 10-12. Preliminary numerical experiments (Szymańska 2012) showed that the BCB model was more suitable than the MVG model to represent the macroscopic air trapping caused by material heterogeneities. Note that due to looser packing of sand in the 2D flume experiments, resulting in larger pores, the real values of the entry pressure can be expected to be smaller than the estimated ones.

- The residual saturations of both water and air are set to zero, which means that the value of the saturated water content was equal to the porosity.

a The relative permeability functions for water and air in each type of sand are assumed to follow the BCB model, Eqs. 8-9.

a The intrinsic permeability of each medium corresponds to the value obtained in the independent experiments (again, due to larger pores, one can expect the real values to be higher).

The parameters based on the above assumptions are listed in Table 1 and denoted as $\mathrm{BCB} 0$ - first estimation. The results of the simulations with $2 \mathrm{PH}$ model for BCB0 parameters are shown in Figs. 4 and 5. Comparing saturation distribution in Fig. 4 with the one observed in the experiments one can note a qualitative agreement regarding the effect of structural trapping, i.e., the upper parts of inclusions remain at low water saturation. However, in the 


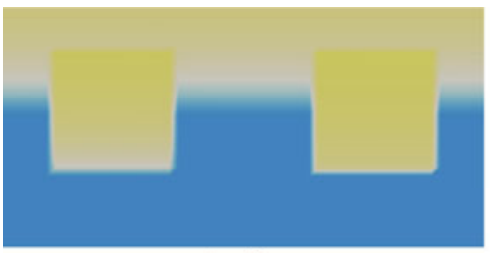

$\mathrm{t}=0$

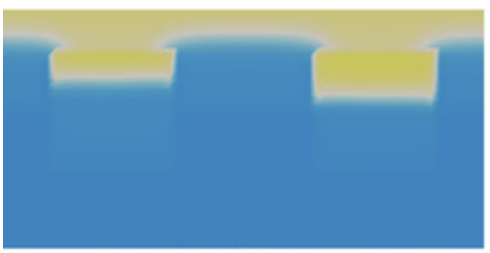

$t=420 s$

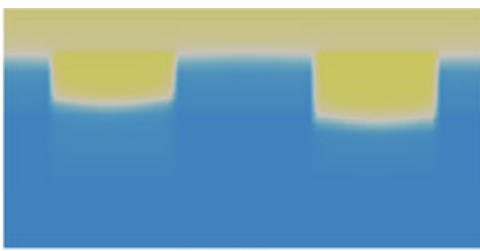

$\mathrm{t}=240 \mathrm{~s}$

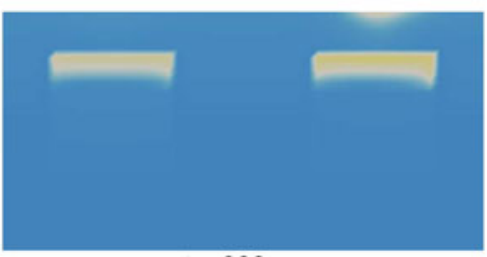

$t=900 s$
1.0

0.8

0.6

0.4

0.2

0.0

Fig. 4. Distribution of water saturation in the flume at four stages of the experiment, simulations with $2 \mathrm{PH}$ model with $\mathrm{BCB} 0$ set of parameters.

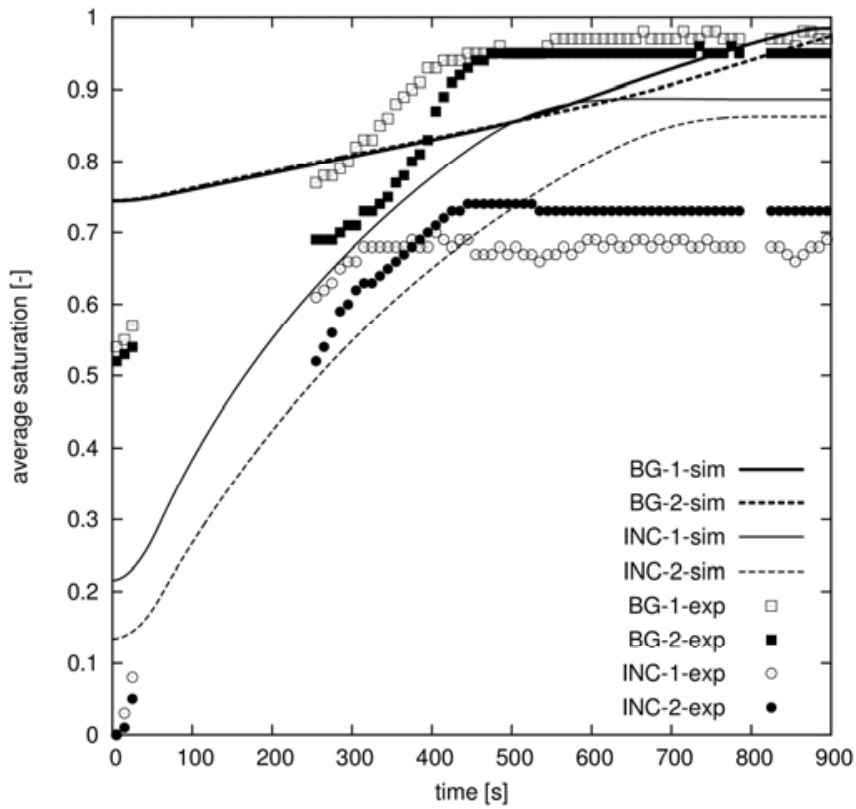

Fig. 5. Evolution of water saturation in four regions of the flume, simulations with $2 \mathrm{PH}$ model with $\mathrm{BCB} 0$ set of parameters.

numerical simulations steady state is not reached even for $t=900 \mathrm{~s}$, as there is still some air flowing out of the background material above the coarse inclusion. Comparison of the evolution of average saturation in each material 


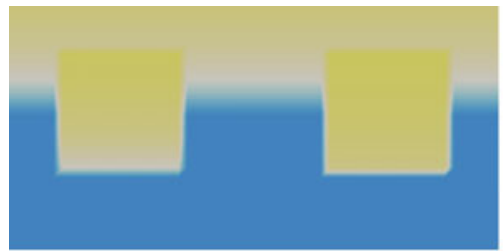

$t=0$

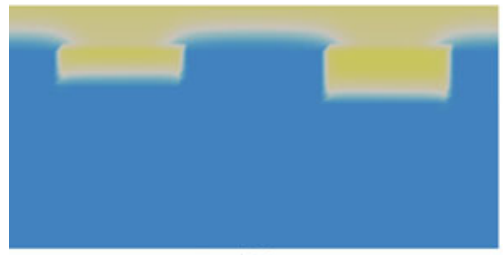

$t=420 s$

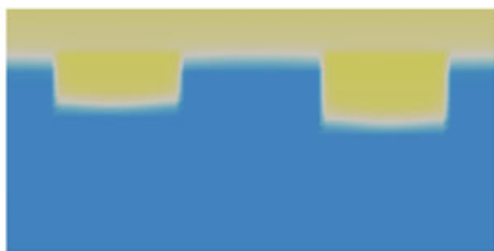

$t=240 s$

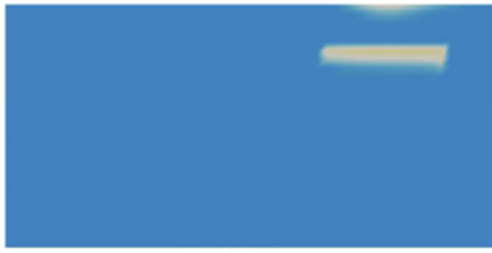

$t=900 s$
1.0

0.8

0.6

0.4

0.2

0.0

Fig. 6. Distribution of water saturation in the flume at four stages of the experiment, simulations with RE model with BCB0 set of parameters.

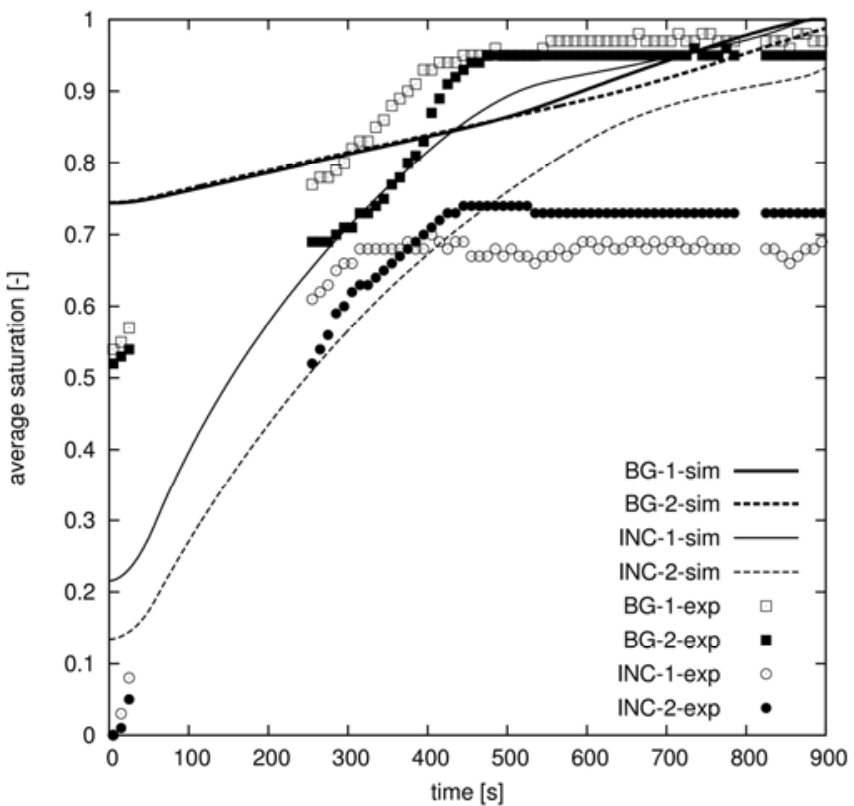

Fig. 7. Evolution of average water saturation in four regions of the flume, simulations with RE model with BCB0 set of parameters.

region (Fig. 5) also shows that the numerical simulations predict changes in the background material saturations long after the development of steady state in the experiments. On the other hand, the saturation in the inclusions 
becomes stable before the end of the simulation, but later than in the experiments. The amount of air trapped in inclusions is smaller according to the simulations, as compared to observations ( $c a .12 \%$ of the pore volume in the simulations versus ca. $30 \%$ in the experiment). Also, BCB0 parameters lead to higher initial saturations in all four regions (by about 15 to $20 \%$ of pore volume). In the fine sand, the simulations predict much slower increase in saturation, compared to the experiment in the initial stage of infiltration. In the medium and coarse sand, the rate of saturation change is similar according to the simulations and experiment.

For the same parameter set BCB0 we also carried out a simulation using $\mathrm{RE}$ and the results are shown in Figs. 6 and 7. For $900 \mathrm{~s}$ the medium sand inclusion is fully saturated, while the coarse sand inclusion is still unsaturated in its upper part, but it is clear that the saturation in the coarse inclusion steadily increases (Fig. 7). For a longer time of about $1200 \mathrm{~s}$ both inclusions become fully saturated (results not shown here). RE does not account for the fact that water saturation can be increased only if there is a possibility for pore air to flow out. Consequently, saturation increases everywhere, even if the cells are fully surrounded with water. However, for the initial phase of infiltration, before the structural air trapping effects occur (at time of about $400 \mathrm{~s}$ ), the results obtained with $2 \mathrm{PH}$ and $\mathrm{RE}$ models are very close to each other.

The major findings from these preliminary simulations were as follows. First, it was clear that the parameters from different experiment do not provide accurate results. This could, however, be expected because of different sand porosity. Second, the Richards equation could not qualitatively correctly capture the trapping effect, while the two phase model was able to do so.

\section{PARAMETER FITTING}

In order to improve the fit between the two-phase model and the experimental results we adjusted the hydraulic parameters by a trial and error procedure, based on the following assumptions:

$\checkmark$ due to larger porosity in each material the permeability should be increased, while the air entry pressure should be decreased in each material;

a parameter $\lambda$ can be increased, since all sands have rather uniform, well sorted grains, which implies a sharp decrease in saturation just above the air entry value;

$\square$ the residual air saturation in all types of sand should be non-zero, i.e., the saturated water content $\theta_{s w}$ should be smaller than the porosity $\phi$; the residual air saturation should be larger in the inclusions than in the background material. 


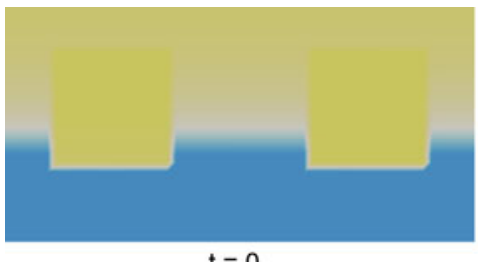

$t=0$

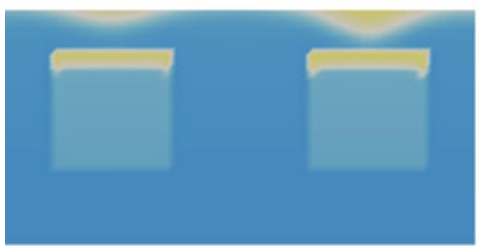

$t=420 s$

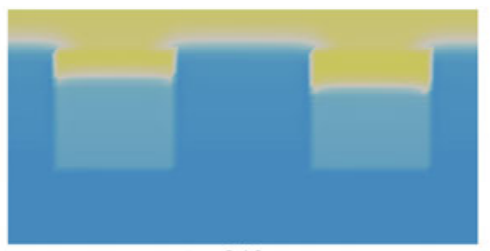

$\mathrm{t}=240 \mathrm{~s}$

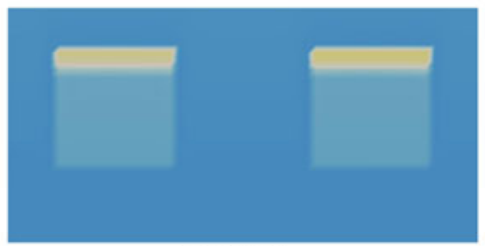

$t=900 s$

Fig. 8. Distribution of water saturation in the flume at four stages of the experiment, simulations with $2 \mathrm{PH}$ model with best-fitted hydraulic parameters $\mathrm{BCB} 1$, including pore-scale trapping.

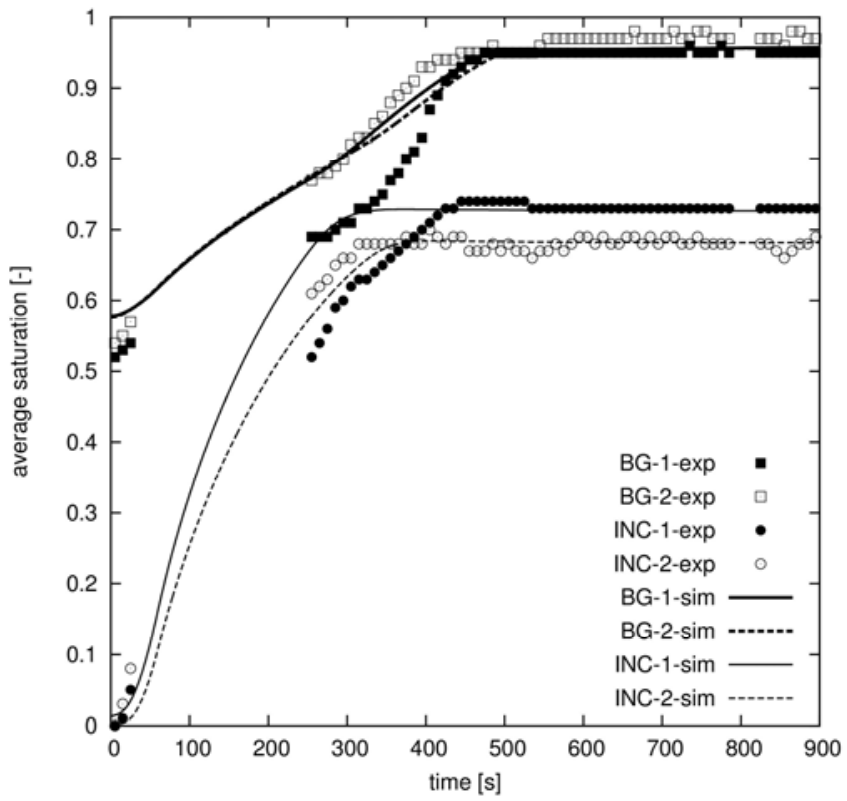

Fig. 9. Evolution of average water saturation in four regions of the flume, simulations with $2 \mathrm{PH}$ model with fitted hydraulic parameters BCB1, including pore-scale trapping.

By performing a large number of numerical tests we arrived at the set of parameters shown in Table 1 as BCB1 - best fit). While these parameters are clearly different from the initial estimation, the discrepancy seems accepta- 


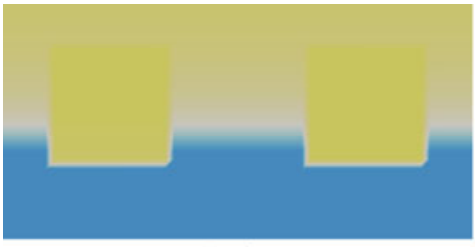

$\mathrm{t}=0$

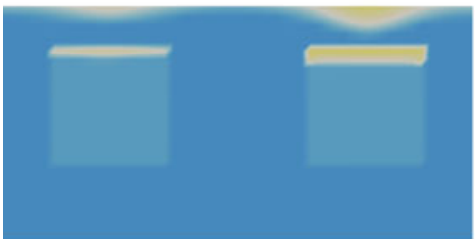

$t=420 s$

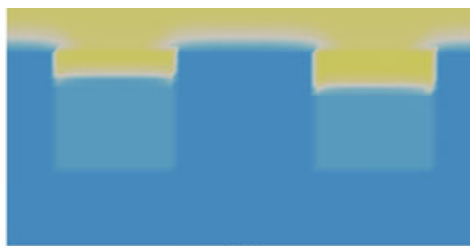

$t=240 s$

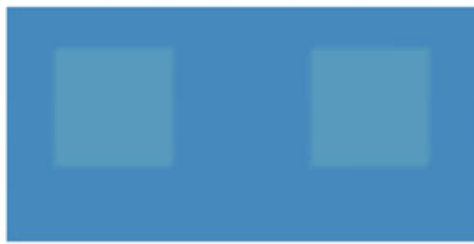

$t=900 s$

Fig. 10. Distribution of water saturation in the flume at four stages of the experiment, simulations with RE model with best-fitted hydraulic parameters BCB1, including pore-scale trapping.

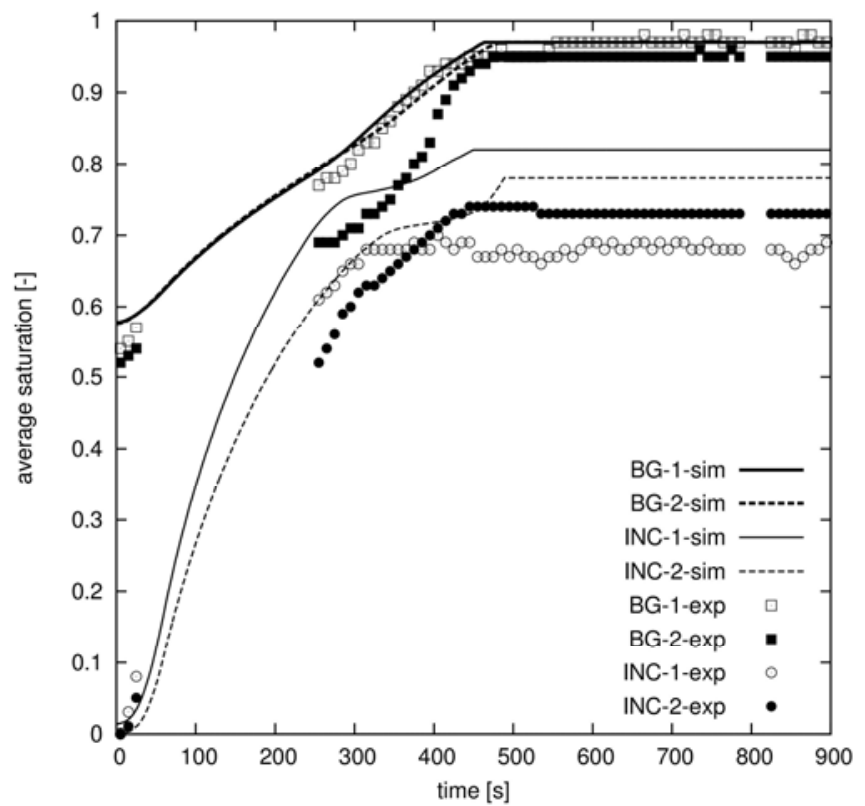

Fig. 11. Evolution of average water saturation in four regions of the flume, simulations with RE model with best-fitted hydraulic parameters BCB1, including porescale trapping.

ble in view of the assumptions listed above. The intrinsic permeability increased about 4 times for the fine sand and about 2 times for the other sands, while the entry pressure decreased by about $30 \%$ for each of the materials. 


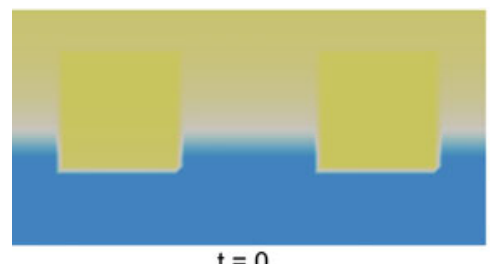

$t=0$

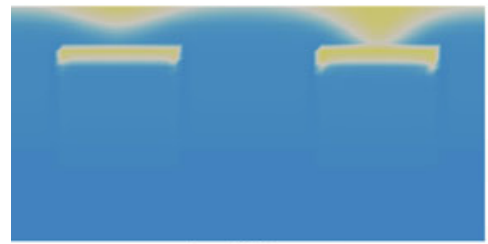

$\mathrm{t}=420 \mathrm{~s}$

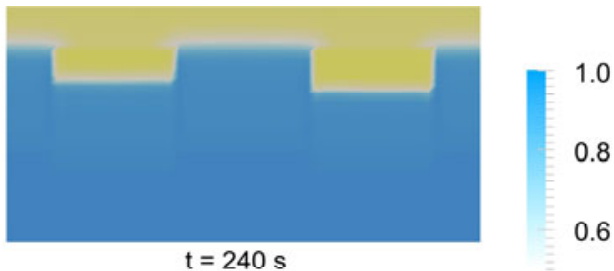

0.4

0.2

0.0

Fig. 12. Distribution of water saturation in the flume at four stages of the experiment, simulations with $2 \mathrm{PH}$ model with best-fitted hydraulic parameters, assuming no pore-scale air trapping.

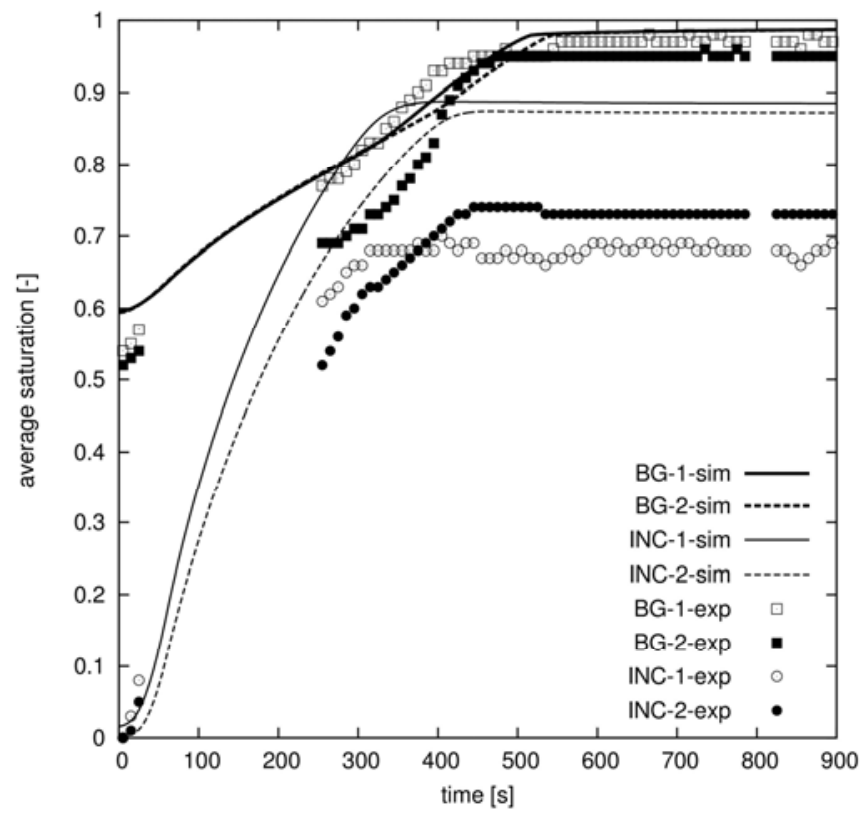

Fig. 13. Evolution of average water saturation in four regions of the flume, simulations with $2 \mathrm{PH}$ model with best-fitted hydraulic parameters, assuming no pore-scale trapping.

The fitted residual air content $\theta_{r a}=\phi-\theta_{s w}$ ranges from 0.015 in the fine material to 0.097 in the coarse material. 
The results of the simulations performed with fitted BCB1 parameters are present in Figs. 8 and 9. They are in a good agreement with the results obtained from experiments. The initial saturation, saturation change rate, time to steady state and steady state saturation are generally well represented. Some discrepancies can be observed for the medium sand inclusion (INC1) and the surrounding fine sand (BG1). In the experiments, BG1 region saturation was significantly lower than $\mathrm{BG} 2$ saturation for time between 200 and $300 \mathrm{~s}$. However, we were not able to represent this effect in any of the tested combinations of parameters. It could be caused by local heterogeneities within the background sand or by non-uniform water supply from the bottom.

The same set of parameters was used in a simulation with RE. The results are presented in Figs. 10 and 11. In this case there is no structural trapping effect and only pore scale air trapping occurs. At the final steady state, each inclusion has a uniform saturation, corresponding to its $\theta_{s w}$ value, which does not agree with the non-uniform distribution of water saturation observed in the experiments. On the other hand, the evolution of average saturation is in a qualitatively good agreement with the experiment, but the final values of water saturation in the inclusions are higher than observed. A better agreement of the saturation curves can be obtained for RE if the residual air saturation for coarse and medium sand is increased (results not shown here). However, it does not affect the final distribution of saturation in inclusions, which is still uniform, in contrast to the experimental results.

In order to further investigate the interplay between structural and pore scale air trapping we performed an additional simulation using $2 \mathrm{PH}$ model with a modified set of parameters BCB1 with residual air saturations set to 0 for each material. The results are shown in Figs. 12 and 13. This set of parameters produced a better fit than $\mathrm{BCB} 0$, but worse than $\mathrm{BCB} 1$. It can be seen that the effect of structural trapping is well represented, with unsaturated zones in the upper parts of inclusions, but the total amount of trapped air is smaller than in the experiments, due to the neglecting of pore scale air trapping. The structural trapping accounts for about $10 \%$ of pore volume in inclusions.

\section{CONCLUSIONS}

The experiment described in this paper provided additional evidence for the importance of local-scale heterogeneities for water and air flow in porous materials. During infiltration a significant amount of air was trapped in the coarse textures inclusions, both in the form of isolated bubbles in pores and larger unsaturated regions in the upper part of each inclusions. These results are in agreement with earlier experiments (Dunn and Silliman 2003, Dunn 2005) and with theoretical and numerical analyses (Szymkiewicz et al. 2012, 
2014). The experimental results were reproduced with numerical simulations based on a two phase flow model with fitted parameters. The values of parameters obtained by the fitting procedure were in the range expected for the material used in the experiments, although they were different from the values obtained from independent measurements. The discrepancy can be explained by different porosity of sand and flow conditions (imbibition versus drainage). A satisfactory agreement with experimental results could be achieved only if both structural air trapping and pore scale air trapping were included in the model. In contrast, the results obtained with the Richards equation did not match the experiment. In RE only the pore scale trapping effect can be included, by reducing the apparent saturated water content with regard to porosity. However, the resulting final distribution of saturation in inclusions was uniform and the presence of unsaturated regions in the upper part of inclusions could not be reproduced, because RE cannot describe air entry barriers well. These macroscopic trapping effects could be mimicked by an apparent residual air saturation in the RE. However, this modeling approach would be quite unsatisfactory, as the parameters are specific to the initial and boundary conditions in the scenario considered and would need to be changed for different conditions. The $2 \mathrm{PH}$ model is able to predict the macroscopic trapping with unique model parameters. These finding seem to be important for modeling of flow and mass transport in the capillary fringe and water table fluctuation zone.

\section{References}

Aubertin, M., E. Cifuentes, S.A. Apithy, B. Bussière, J. Molson, and R.P. Chapuis (2009), Analyses of water diversion along inclined covers with capillary barrier effects, Can. Geotech. J. 46, 10, 1146-1164, DOI: 10.1139/T09-050.

Berkowitz, B., S.E. Silliman, and A.M. Dunn (2004), Impact of the capillary fringe on local flow, chemical migration and microbiology, Vadose Zone J. 3, 2, 534-548.

Bogacz, P., J. Kaczmarek, and D. Leśniewska (2006), Influence of air entrapment on flood embankment failure mechanics - model tests, Technol. Sci. 11, 188201.

Brooks, R.H., and A.T. Corey (1964), Hydraulic properties of porous media, Technical Report, Hydrology Paper 3, Colorado State University, Fort Collins, Colorado, USA.

Burdine, N.T. (1953), Relative permeability calculations from pore size distribution data, J. Petrol. Technol. 5, 03, 71-78. 
de Neef, M.J., and J. Molenaar (1997), Analysis of DNAPL infiltration in a medium with a low permeable lens, Comput. Geosci. 1, 2, 191-214, DOI: 10.1023/ A:1011569329088.

Delfs, J.O., W. Wang, T. Kalbacher, A.K. Singh, and O. Kolditz (2013), A coupled surface/subsurface flow model accounting for air entrapment and air pressure counterflow, Environ. Earth Sci. 69, 2, 395-414, DOI: 10.1007/ s12665-013-2420-1.

Dunn, A.M. (2005), Air and LNAPL entrapment in the partially saturated fringe: Laboratory and numerical investigations, Ph.D. Thesis, University of Notre Dame, Indiana, USA.

Dunn, A.M., and S.E. Silliman (2003), Air and water entrapment in the vicinity of the water table, Ground Water 41, 729-734.

Forsyth, P.A. (1988), Comparison of the single-phase and two-phase numerical model formulation for saturated-unsaturated groundwater flow, Comput. Meth. Appl. Mech. Eng. 69, 2, 243-259, DOI: 10.1016/0045-7825(88) 90190-9.

Haberer, C.M., M. Rolle, O.A. Cirpka, and P. Grathwohl (2015), Impact of heterogeneity on oxygen transfer in a fluctuating capillary fringe, Ground Water 53, 1, 57-70, DOI: 10.1111/gwat.12149.

Hammecker, C., A.C.D. Antonino, J.L. Maeght, and P. Boivin (2003), Experimental and numerical study of water flow in soil under irrigation in northern senegal: evidence of air entrapment, Europ. J. Soil Sci. 54, 3, 491-503, DOI: 10.1046/j.1365-2389.2003.00482.x.

Helmig, R. (1997), Multiphase Flow and Transport Processes in the Subsurface: A Contribution to the Modeling of Hydrosystems, Springer.

Kuang, X., J.J. Jiao, L. Wan, X. Wang, and D. Mao (2011), Air and water flows in a vertical sand column, Water Resour. Res. 47 4, W04506, DOI: 10.1029/ 2009WR009030.

Lenhard, R.J., J.C. Parker, and S. Mishra (1989), On the correspondence between Brooks-Corey and van Genuchten models, J. Irrig. Drain. Eng. ASCE 115, 4, 744-751, DOI: 10.1061/(ASCE)0733-9437(1989)115:4(744).

Leśniewska, D., H. Zaradny, P. Bogacz, and J. Kaczmarek (2008), Study of flood embankment behaviour induced by air entrapment. In: P. Samuels, S. Huntington, W. Allsop, and J. Harrop (eds.), Flood risk Management: Research and Practice, Taylor \& Francis, London, 655-665.

Likos, W.J., N. Lu, and J.W. Godt (2014), Hysteresis and uncertainty in soil waterretention curve parameters, J. Geotech. Geoenviron. Eng. 140, 4, 04013050, DOI: 10.1061/(ASCE)GT.1943-5606.0001071.

Luckner, L., M.Th. van Genuchten, and D.R. Nielsen (1989), A consistent set of parametric models for the two-phase flow of immiscible fluids in the subsurface, Water Resour. Res. 25, 10, 2113-2124, DOI: 10.1029/ WR025i010p02187. 
Marinas, M., J.W. Roy, and J.E. Smith (2013), Changes in entrapped gas content and hydraulic conductivity with pressure, Ground Water 51, 1, 41-50, DOI: 10.1111/j.1745-6584.2012.00915.x.

McLeod, H.C., J.W. Roy, and J.E. Smith (2015), Patterns of entrapped air dissolution in a two-dimensional pilot-scale synthetic aquifer, Ground Water 53, 2, 271-281, DOI: 10.1111/gwat.12203.

Mikelic, A., C.J. van Duijn, and I.S. Pop (2002), Effective equations for two-phase flow with trapping on the micro scale, SIAM J. Appl. Math. 62, 5, 15311568, DOI: $10.1137 /$ S0036139901385564.

Mualem, Y. (1976), A new model for predicting the hydraulic conductivity of unsaturated porous media, Water Resour. Res. 12, 3, 513-522, DOI: 10.1029/ WR012i003p00513.

Oldenburg, C.M., and K. Pruess (1993), On numerical modeling of capillary barriers, Water Resour. Res. 29, 4, 1045-1056, DOI: 10.1029/92WR02875.

Prédélus, D., A.P. Coutinho, L. Lassabatere, B. Bien Le, T. Winiarski, and R. Angulo-Jaramillo (2015), Combined effect of capillary barrier and layered slope on water, solute and nanoparticle transfer in an unsaturated soil at lysimeter scale, J. Contam. Hydrol. 181, 69-81, DOI: 10.1016/j.jconhyd. 2015.06.008.

Richards, L.A. (1931), Capillary conduction of liquids through porous medium, J. Appl. Physics. 1, 318-333, DOI: 10.1063/1.1745010.

Saadatpoor, E., S.L. Bryant, and K. Sepehrnoori (2009), Effect of capillary heterogeneity on buoyant plumes: a new local mechanism, Energy Procedia 1, 1, 3299-3306, DOI: 10.1016/j.egypro.2009.02.116.

Schweizer, B. (2008), Homogenization of degenerate two-phase flow equations with oil-trapping, SIAM J. Math. Anal. 39, 1740-1763, DOI: 10.1137/ 060675472.

Silliman, S.E., B. Berkowitz, J. Simunek, and M.Th. Van Genuchten (2002), Fluid flow and solute migration within the capillary fringe, Ground Water 40, 1, 76-84, DOI: 10.1111/j.1745-6584.2002.tb02493.x.

Szymańska, P. (2012), Flow in unsaturated porous media: Numerical and experimental evaluation of the two-phase model and the richards equation, M.Sc. Thesis, Gdańsk University of Technology, Gdańsk, Poland.

Szymkiewicz, A. (2013), Modelling Water Flow in Unsaturated Porous Media: Accounting for Nonlinear Permeability and Material Heterogeneity, GeoPlanet: Earth and Planetary Sciences, Springer.

Szymkiewicz, A., R. Helmig, and H. Kuhnke (2011), Two-phase flow in heterogeneous porous media with non-wetting phase trapping, Transport Porous Med. 86, 1, 27-47, DOI: 10.1007/s11242-010-9604.

Szymkiewicz, A., R. Helmig, and I. Neuweiler (2012), Upscaling unsaturated flow in binary porous media with air entry pressure effects, Water Resour. Res. 48, 4, W04522, DOI: 10.1029/2011WR010893. 
Szymkiewicz, A., I. Neuweiler, and R. Helmig (2014), Influence of heterogeneous air entry pressure on large scale unsaturated flow in porous media, Acta Geophys. 62, 5, 1179-1191, DOI: 10.2478/s11600-014-0224-7.

Tegnander, C. (2001), Models for groundwater flow: A numerical comparison between Richards model and fractional flow model, Transport Porous Med. 43, 2, 213-224, DOI: 10.1023/A:1010749708294.

Touma, J., and M. Vauclin (1986), Experimental and numerical analysis of twophase infiltration in a partially saturated soil, Transport Porous Med, 1, 1, 27-55, DOI: 10.1007/BF01036524.

Touma, J., G. Vachaud, and J.-Y. Parlange (1984), Air and water flow in a sealed, ponded vertical soil column: experiment and model, Soil Science 137, 3, 181-187.

Vachaud, G., M. Vauclin, D. Khanji, and M. Wakil (1973), Effects of air pressure on water flow in an unsaturated stratified vertical column of sand, Water Resour. Res. 9, 1, 160-173, DOI: 10.1029/WR009i001p00160.

van Duijn, C.J., H. Eichel, R. Helmig, and I.S. Pop (2007), Effective equations for two-phase flow in porous media: the effect of trapping at the micro-scale, Transport Porous Med. 69, 3, 411-428, DOI: 10.1007/s11242-006-9089-9.

van Genuchten, M.Th. (1980), A closed form equation for predicting the hydraulic conductivity of unsaturated soils, Soil Sci. Soc. Am. J. 44, 5, 892-898, DOI: 10.2136/sssaj1980.03615995004400050002x.

Vasin, M., P. Lehmann, A. Kaestner, R. Hassanein, W. Nowak, R. Helmig, and I. Neuweiler (2008), Drainage in heterogeneous sand columns with different geometric structures, Adv. Water Resour. 31, 9, 1205-1220, DOI: 10.1016/j.advwatres.2008.01.004.

Webb, S.W. (1998), Using TOUGH2 to model capillary barriers. In: Proc. TOUGH Workshop 1998, 4-6 May 1998, Lawrence Berkeley National Laboratory, Berkeley, California, USA.

Yakirevich, A., T.J. Gish, J. Simunek, M.Th. Van Genuchten, Y.A. Pachepsky, T.J. Nicholson, and R.E. Cady (2010), Potential impact of a seepage face on solute transport to a pumping well, Vadose Zone J. 9, 3, 686-696, DOI: $10.2136 /$ vzj2009.0054. 